\title{
Urinary System Findings Specimen Type
}

National Cancer Institute

\section{Source}

National Cancer Institute. Urinary System Findings Specimen Type. NCI Thesaurus. Code C162352.

The type of material sample used for urinary system testing. 